\title{
Erratum to: Survivin-mediated Therapeutic Efficacy of Gemcitabine through Glucose-regulated Protein 78 in Hepatocellular Carcinoma
}

\author{
Chin-Sheng Hung, MD ${ }^{1,2,3,4}$, Shen-Fu Lin, MS ${ }^{8}$, Hui-Hsiung Liu, MD, PhD ${ }^{7}$, Li-Jen Kuo, MD ${ }^{1,2,3,4}$, Li-Tzu Li, PhD ${ }^{6}$, \\ Hou-Yu Su, MD ${ }^{1,5}$, Phui-Ly Liew, MD ${ }^{9}$, Feng-Yen Lin, PhD ${ }^{10,11}$, Po-Li Wei, MD, PhD ${ }^{1,2,3,4}$, Der-Zen Liu, PhD ${ }^{8}$, and \\ Yu-Jia Chang, $\mathbf{P h D}^{1,2,3,4}$ \\ ${ }^{1}$ Graduate Institute of Clinical Medicine, College of Medicine, Taipei Medical University, Taipei, Taiwan; ${ }^{2}$ Department of \\ Surgery, School of Medicine, College of Medicine, Taipei Medical University, Taipei, Taiwan; ${ }^{3}$ Division of General \\ Surgery, Department of Surgery, Taipei Medical University Hospital, Taipei Medical University, Taipei, Taiwan; ${ }^{4}$ Cancer \\ Research Center, Taipei Medical University Hospital, Taipei Medical University, Taipei, Taiwan; ${ }^{5}$ Department of Surgery, \\ Saint Mary's Hospital Luodong, Luodong, Yilan, Taiwan; ${ }^{6}$ Imaging Core Facilities, Institute of Cellular and Organismic \\ Biology, Academia Sinica, Taipei, Taiwan; ${ }^{7}$ Department of Public Health, School of Public Health, Taipei Medical \\ University, Taipei, Taiwan; ${ }^{8}$ Graduate Institute of Biomedical Materials and Engineering, Taipei Medical University, \\ Taipei, Taiwan; ${ }^{9}$ Department of Pathology, Shuang-Ho Hospital, Taipei Medical University, Taipei, Taiwan; ${ }^{10}$ Department \\ of Internal Medicine, School of Medicine, College of Medicine, Taipei Medical University, Taipei, Taiwan; ${ }^{11}$ Division of \\ Cardiology, Taipei Medical University Hospital, Taipei, Taiwan
}

ERRATUM TO: ANN SURG ONCOL (2012) 19:2744-2752

DOI 10.1245/S10434-011-2188-Z

Figure 1 was incorrect in the original article. The correct figure is as follows:

The online version of the original article can be found under doi:10. 1245/s10434-011-2188-z.

(C) Society of Surgical Oncology 2013

Published Online: 4 December 2013

Y.-J. Chang, $\mathrm{PhD}$

e-mail: r5424012@tmu.edu.tw 


\section{(A) Mahlavu cells}

Control survivinKD
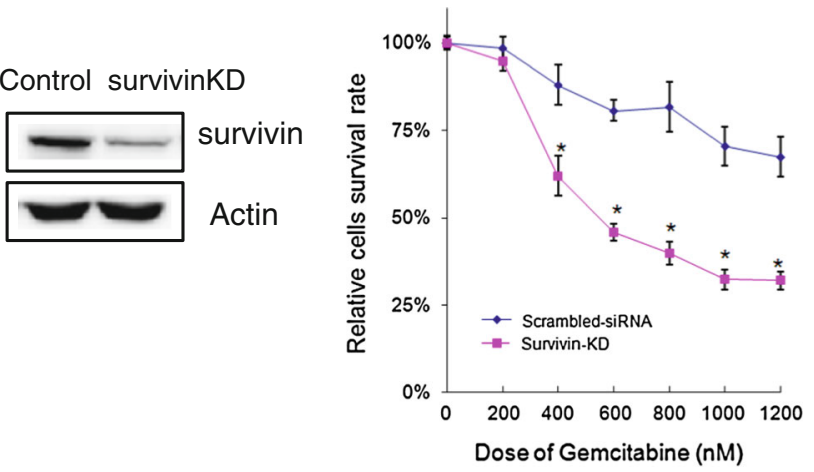

(B) $\quad \underline{\text { HepJ5 }}$

\section{Control survivinKD}
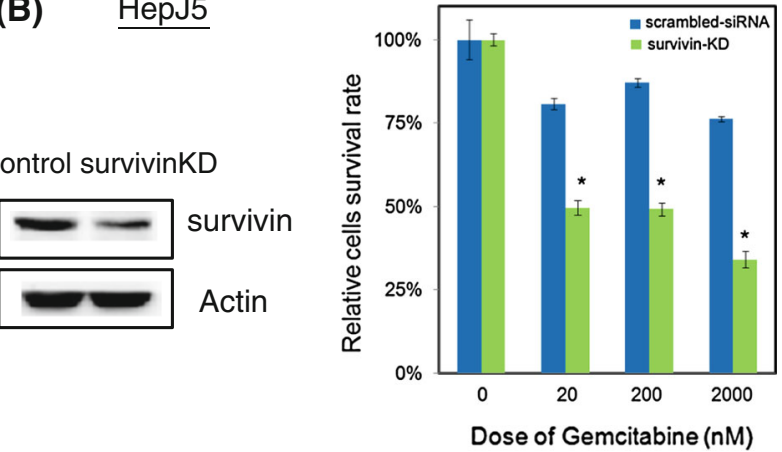

FIG. 1 The percentages of the viable HepJ5 and Mahlavu cells after treated with gemcitabine. a The survivin-KD and scrambled control Mahlavu cells $\left(2 \times 10^{4}\right.$ cells/well $)$ were plated into 24 -well plates with various dose of gemcitabine for 48 hours. The population survival cells were determined by MTT assay. b The survivin-KD and scrambled control HepJ5 cells were treated with various dose of gemcitabine for 48 hours. The population survival cells were determined by MTT assay. All the experiments were repeated at least 3 times independently. $* P<0.05$ 